\title{
The value of choice: development of a new measurement tool
}

\author{
Jane Ogden, Emma Daniells and Julie Barnett
}

\author{
ABSTRACT \\ Background \\ Current policy advocates individual choice across a \\ number of domains, including healthcare provision. \\ Aim \\ To develop a new tool for measuring people's beliefs \\ about the value of choice in the context of healthcare \\ provision. \\ Design of study \\ Cross-sectional survey. \\ Setting \\ Four general practices in South East England.

\section{Method}

Nine items relating to health and general domains, derived from in-depth interviews with 22 participants, were collated into a questionnaire. This formed the measurement tool called the Choice Questionnaire (ChQ), which was completed by 823 consecutive patients (response rate $=81.2 \%$ ).

\section{Results}

Principal components analysis resulted in two factors: having choices (for example, 'I like to know all the possible ways in which I could be treated') and making choices (for example, 'I am happy for the doctor to make decisions for me'). These two constructs showed good internal consistency. One item was deleted, resulting in the 8-item ChQ. Beliefs about choice in health and general domains were not discrete. The vast majority of participants endorsed having choices as positive. In contrast, beliefs about the value of making choice were more mixed. A greater endorsement of both these aspects of choice was related to higher educational status.

\section{Conclusion}

Results from this study indicate that choice can be usefully conceptualised as consisting of two separate constructs: having choices and making choices. The 8-item ChQ has an interpretable factor structure and has been shown to be reliable. It could be used in research to assess beliefs about the value of choice or in the clinical setting to establish whether a patient would prefer to be provided with options or to be managed in a more paternalistic way.

\section{Keywords}

beliefs; choice behaviour; health care; questionnaire design.

\section{INTRODUCTION}

At the turn of this century, ex-Prime Minister Tony Blair and US President George Bush emphasised choice as a central component to reform. In line with this, Schwartz argued that choice has become a core component of modernisation. ${ }^{1}$ Furthermore, it has also penetrated the world of healthcare provision, with patients being referred to as 'consumers', and the production of the Patient's Charter, which emphasises each patients' right to choose how their health is managed. There is also focus on factors such as shared decision making, patient participation, and patient centredness. ${ }^{2}$

Central to this shift is the belief that choice is desirable, and economists and policy makers have conceptualised choice as 'something that one can't have too much of, like clean air or beauty'. ${ }^{3}$ The psychological literature also highlights the positive consequences of choice, indicating that greater choice increases intrinsic motivation, task performance, life skills, and higher outcome evaluations. ${ }^{4-8}$ Such benefits seem to occur regardless of whether choice is actual, trivial, or illusory. ${ }^{9}$

Researchers have also argued that individuals systematically prefer to take the choice option rather than the 'no-choice option'; this has been conceptualised as reflecting a need to keep their options open and has been labelled 'the lure of choice'..$^{10}$ In contrast, other research demonstrates how choice can have negative consequences. In The Paradox of Choice, Schwartz describes how the proliferation of goods has resulted in people feeling

J Ogden, PhD, professor in health psychology; E Daniells, $\mathrm{MSc}$, research assistant; J Barnett, $\mathrm{PhD}$, senior research fellow, Department of Psychology, University of Surrey, Guildford.

Address for correspondence

Jane Ogden, Department of Psychology, University of

Surrey, Guildford, GU2 7XH.

E-mail: J.Ogden@surrey.ac.uk

Submitted: 9 December 2007; Editor's response: 4 March 2008; final acceptance: 26 March 2008.

(c)British Journal of General Practice.

This article was originally online first. Cite this article as: Br J Gen Pract 2008; 58: 614-618. Advance online publication. DOI: $10.3399 /$ bjgp08X330735 
anxious and overwhelmed when making daily choices. ${ }^{1}$

Using an experimental design, lyengar and Lepper explored the impact of manipulating the degree of choice and concluded that participants' levels of motivation and satisfaction were lower following extensive, rather than limited, choices. ${ }^{9}$ This research team also concluded from their experimental studies that the benefits of choice may be culturally specific. ${ }^{11}$ Botti and lyengar suggested that, within some cultures, choice only confers benefit to the chooser if the person constraining the choice is respected and deemed trustworthy. ${ }^{4}$

Choice is central to policy and healthcare provision and has become incorporated into the ways in which individual motivations and expectations are conceptualised. However, there is no reliable measure of the extent to which individuals value choice in their day-to-day lives. The present study aimed to develop and assess the reliability of a measure of beliefs about the value of choice; assess the extent to which choice is considered desirable; and examine the role of demographic variables in predicting any variation in this belief. The study also aimed to explore whether beliefs about choice were domain specific and whether placing a high value on choice in general was reflected in a greater endorsement of choice in the context of healthcare provision.

\section{METHOD \\ Design}

The study involved a large-scale, cross-sectional survey of patients in primary care.

\section{Developing the measure}

In-depth qualitative interviews were carried out with 22 people who responded to advertisements at the University of Surrey. Interviewers sought to determine their beliefs about choice across a number of domains including health care, shopping, eating out, and relationships. Participants in this study described choice in terms of a number of key themes including the value of choice, trust in the choice provider, the appearance of choice rather than the substance of any real choice, and regret following choice. Detailed results from this study are being published elsewhere. ${ }^{12,13}$ For the purpose of the present study, nine items were selected that reflected the range of views participants held about choice. In order that the questionnaire could be used in the health domain and from a more general perspective, four items were selected to reflect beliefs about choice in general and five were selected to reflect beliefs about choice in the context of health care.

\section{How this fits in}

Current policy and research emphasise the importance of choice in the healthcare context but, to date, there is no measurement tool to assess patients' beliefs about the value of choice. This article presents a new scale to assess beliefs about choice. Choice is best conceptualised as consisting of beliefs about having choices and making choices.

Participants were asked to rate the extent to which they agreed with each statement using a five-point Likert scale ranging from (1) 'strongly disagree' to (5) 'strongly agree'. In addition, all participants were asked to record their age, sex, ethnicity (white, black, Asian, or other), educational level (none, GCSEs, Alevels, degree and above), and how many times they had visited their doctor in the past year $(0,1-3,4-8$, 9-12 consultations).

They were also asked to rate their own current health (self-reported health status) using two scales: one scale ranged from (1) 'worst possible' to (5) 'best possible' health, the other included health ratings of poor, good, very good, and excellent health. These two items were summated to create a mean selfreported health score. For descriptive purposes, scores were categorised into low, medium, and high self-reported health status.

\section{Table 1. Participants' demographics ${ }^{a}$}

\begin{tabular}{|c|c|}
\hline \multicolumn{2}{|l|}{ Age, years } \\
\hline Mean & 40.02 \\
\hline SD & 17.54 \\
\hline Range & $18-89$ \\
\hline \multicolumn{2}{|l|}{ Sex, $n(\%)$} \\
\hline Male & $263(32.5)$ \\
\hline Female & $546(67.5)$ \\
\hline \multicolumn{2}{|l|}{ Ethnicity, $n(\%)$} \\
\hline White & $717(88.6)$ \\
\hline Black & $24(3.0)$ \\
\hline Asian & $39(4.8)$ \\
\hline Other & $29(3.6)$ \\
\hline \multicolumn{2}{|l|}{ Education, $n(\%)$} \\
\hline None & $134(17.7)$ \\
\hline GCSEs or equivalent & $122(16.1)$ \\
\hline A-levels or equivalent & $194(25.6)$ \\
\hline Degree or above & $309(40.7)$ \\
\hline \multicolumn{2}{|c|}{ Self-reported health status, $n(\%)$} \\
\hline Low & $277(35.7)$ \\
\hline Medium & $192(24.7)$ \\
\hline High & 307 (39.6) \\
\hline \multicolumn{2}{|c|}{ Number of visits to GP in past year, $n(\%)$} \\
\hline None & $48(6.1)$ \\
\hline $1-3$ & $366(46.4)$ \\
\hline $4-8$ & $264(33.5)$ \\
\hline $9-12+$ & $110(14.0)$ \\
\hline
\end{tabular}

${ }^{\mathrm{a}}$ Total number of participants $=823$; number of responders differed slightly between variables. 


\section{Table 2. Factor structure of preliminary scale.}

Factor $1^{a}$

Factor $2^{b}$

(32.0\% of variance) ( $16.1 \%$ of variance)

General items

I am the kind of person who likes to be offered

choices, rather than being told the best way forward

I am the kind of person who is happy to be told

$0.57^{\circ}$

$-0.35$

what the best options are ${ }^{d}$

I am the kind of person who feels overwhelmed by

choice and would rather it could be more simple

$0.42^{\circ}$

$0.49^{c}$

$-0.21$

$0.66^{c}$

I prefer to know what options are available to me

$0.82^{\circ}$

$-0.12$

Health items

I like to know all the possible ways in which I

could be treated

I am not interested in finding out what all the options are for treating my problem

I am happy for the doctor to make decisions for me

It's not important to me to make my own

healthcare decisions

I prefer to make my own mind up about what treatment I will have

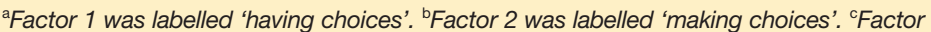
loading $>0.4$. ' $/$ tem deleted from analysis as it did not differentiate between the two factors.

\section{Participants}

Following NHS ethics committee approval, questionnaires were handed out to consecutive patients attending four general practices in Surrey. Practices were selected to provide a sample of patients who varied in terms of socioeconomic status.

Patients were asked if they would like to fill in a short questionnaire concerning their beliefs about choice. They were then asked to consider the items on the questionnaire in general; they were not asked to relate them to the problem they were attending for on that day. Patients were excluded if they were under 18 years of age or did not speak adequate English.

Of the 1031 patients who were asked to complete the questionnaire, 893 agreed. A total of 843 questionnaires were returned, giving a response rate of $81.2 \%$. Of these 843,20 questionnaires were discarded due to being incomplete.

\section{Data analysis}

Data were analysed to determine participants' demographic characteristics and to explore the factor structure of the scale using principal components analysis with varimax rotation, which explores how the different items relate to each other. They were then analysed to ascertain the distribution of beliefs about choice using the new scale, to assess its reliability using Cronbach's alpha, and to assess the role of demographics in predicting the resulting factors using multiple regression analysis.

\section{RESULTS}

\section{Sample characteristics}

Participants' demographics are shown in Table 1. Most participants were women, white, and educated up to degree level with a mean age of 40 years. Just over one-third also had a university degree. Self-reported health status was split fairly evenly across the low, medium, and high categories and nearly half of the participants stated that they had been to their GP between one and three times in the past year.

\section{Factor structure}

Principal component analysis was carried out with varimax rotation which produced two factors (Table 2). These factors were produced after rotation with eigenvalues greater than one, which was selected as the cut-off point and was also supported by the scree plot. Factor 1 had an eigenvalue of 2.9 and accounted for $32.0 \%$ of the variance, and Factor 2 had an eigenvalue of 1.5 and accounted for $16.1 \%$ of the variance.

In line with statistical recommendations, the factor loadings ( $>0.4$ ) indicated that Factor 1 consisted of four items and was conceptualised as having choices, as these items all related to the availability of a number of choices relating to both health and non-health domains. Factor 2 also consisted of four items and was conceptualised as making choices, as all items described the notion of resolution and of choices being made. One item ('I am the kind of person who is happy to be told what the best options are') loaded onto both factors and was deleted from the analysis as it did not differentiate between the two factors. Therefore, the final questionnaire consisted of eight items describing two constructs, which were labelled 'having choices' and 'making choices'. The items relating to choice in general, and healthcare provision in particular, did not load onto separate factors and were distributed across the having-choice and making-choice factors.

\section{Determining beliefs about choice}

Results were analysed to determine beliefs about choice in terms of the individual items and total choice scores. Five-point Likert scores were recoded into three categories (no, not sure, yes); results are shown in Table 3. Most participants endorsed the benefits of having choices available to them in general as well as in terms of health, whether judging by the individual items or the total having-choice score. In contrast, the results for the items relating to making choices were more varied and, for the most part, more negative. In particular, although one-third of participants were positive 
Table 3. Participants' beliefs about choice using the 8-item Choice Questionnaire, $n$ (\%).

\begin{tabular}{|c|c|c|c|}
\hline & $\begin{array}{c}\text { No: } \\
1-2 \text { on } \\
\text { Likert scale }\end{array}$ & $\begin{array}{c}\text { Not sure: } \\
3 \text { on } \\
\text { Likert scale }\end{array}$ & $\begin{array}{c}\text { Yes: } \\
4-5 \text { on } \\
\text { Likert scale }\end{array}$ \\
\hline \multicolumn{4}{|l|}{ Having choices } \\
\hline $\begin{array}{l}\text { I am the kind of person who likes to be offered choices } \\
\text { rather than being told the best way forward }\end{array}$ & $68(8.3)$ & $112(13.7)$ & $640(78.0)$ \\
\hline I prefer to know what options are available to me & $15(1.8)$ & $34(4.1)$ & $771(93.7)$ \\
\hline I like to know all the possible ways in which I could be treated & $24(2.9)$ & $36(4.1)$ & $757(92.7)$ \\
\hline $\begin{array}{l}\text { I am not interested in finding out what all the options are } \\
\text { for treating my problem }\end{array}$ & $705(86.8)$ & $43(5.3)$ & $70(8.6)$ \\
\hline Total having-choice score ${ }^{a}$ & $16(2.0)$ & $107(29.6)$ & $787(65.4)$ \\
\hline \multicolumn{4}{|l|}{ Making choices } \\
\hline I am happy for the doctor to make decisions for me & $342(41.9)$ & $250(30.6)$ & $224(27.5)$ \\
\hline It's not important to me to make my own healthcare decisions & $621(75.7)$ & $94(11.5)$ & $105(12.8)$ \\
\hline $\begin{array}{l}\text { I prefer to make my own mind up about what treatment I } \\
\text { will have }\end{array}$ & $126(15.5)$ & $231(28.4)$ & $457(56.1)$ \\
\hline $\begin{array}{l}\text { I am the kind of person who feels overwhelmed by choice and } \\
\text { would rather it could be more simple }\end{array}$ & $446(54.6)$ & $165(20.2)$ & $206(25.2)$ \\
\hline Total making-choice score ${ }^{a}$ & $37(4.5)$ & $521(62.9)$ & $249(30.3)$ \\
\hline Total choice score ${ }^{a}$ & $5(6.3)$ & $213(29.0)$ & $576(72.5)$ \\
\hline
\end{tabular}

about making choices, the remaining two-thirds were either unsure or negative about the importance of making their own choices in both health and general domains.

\section{Internal reliability}

Four of the items were reverse scored so that a higher score consistently reflected a greater endorsement of having choices and making choices. The reliability of these two constructs was then assessed using Cronbach's alpha. The alpha for having choices (four items) was 0.7 and the alpha for making choices (four items) was 0.6. This suggests that both constructs were adequately reliable. There was also a significant positive correlation between the having-choices and making-choices scores $(r=0.440, n=794$, $P<0.0005)$.

\section{Predicting beliefs about choice}

Results were analysed to assess the role of demographic factors (age, sex, ethnicity, education, current health status, and annual visits to doctor) in predicting beliefs about having choices, making choices, and the total choice score using multiple regression analysis. Demographics were entered as independent variables, and beliefs about choice were entered separately as the dependent variables.

Results showed that educational level was the only significant predictor of all three aspects of choice, accounting for $1.7 \%$ of the variance for having choices, $3.1 \%$ of the variance for making choices, and $3.0 \%$ of the variance for the total choice scores. A higher level of education was associated with a greater endorsement of all aspects of choice, although the amount of variance accounted for remained low (Table 4).

\section{DISCUSSION}

\section{Summary of main findings}

The present study aimed to develop a new measure of beliefs about choice. The resulting tool, the 8-item ChQ, was deemed to have good internal consistency and comprised two factors: having choices and making choices.

Previous policy and research papers have highlighted the importance of choice within the area of health as well as more general domains; this study indicates that beliefs about choice are not domain specific and that beliefs about health choices are closely aligned to more general beliefs. However, results also indicate that choice is not a unidimensional construct and can be more usefully conceptualised as relating to two different aspects of choice, namely having choices and making choices. Having choices relates to the availability of a number of options from which an individual can chose, while making choices relates to resolution and the desire for the choice to be made.

This study also explored whether choice is deemed desirable and found that participants consistently endorsed having choices as beneficial, particularly those who had been educated to a higher level (degree level and beyond). In contrast, beliefs about making choices were less consistently 
Table 4. Predicting beliefs about choice.

\begin{tabular}{lccc} 
Variable & $\begin{array}{c}\text { Having choices, } \\
\text { standardised } B \\
(P \text {-value })\end{array}$ & $\begin{array}{c}\text { Making choices, } \\
\text { standardised } B \\
(P \text {-value })\end{array}$ & $\begin{array}{c}\text { Total choice score, } \\
\text { standardised } B \\
(P \text {-value })\end{array}$ \\
\hline Age & $-0.02(0.6)$ & $0.1(0.01)$ & $0.04(0.28)$ \\
\hline Sex & $-0.005(0.9)$ & $-0.05(0.19)$ & $-0.03(0.43)$ \\
\hline Ethnicity & $-0.04(0.3)$ & $-0.06(0.12)$ & $-0.06(0.15)$ \\
\hline Education & $0.13(0.001)$ & $0.14(0.001)$ & $0.16(0.0001)$ \\
\hline Health status & $0.04(0.3)$ & $0.05(0.24)$ & $0.06(0.16)$ \\
\hline Visits to GP & $0.05(0.3)$ & $-0.04(0.4)$ & $0.01(0.8)$ \\
\hline
\end{tabular}

$P \leq 0.0005$.

\section{Implications for future research and clinical practice}

Although people may show consistently positive beliefs about the value of having choices, their beliefs are more varied when they focus on making choices. Such beliefs can be measured using this newly developed tool, the 8-item ChQ, which has a robust factor structure and good internal consistency.

The 8-item ChQ could be applied to future research exploring beliefs about choice. It could also be used in the clinical setting to determine whether individuals prefer to be regarded as 'consumers', in line with current thinking, or whether a more paternalistic approach to their health care would be more appropriate. endorsed the need to make choices for most of the items, the remaining two-thirds were unsure or negative about the need for a choice to be made. Interestingly, the majority disagreed with the statement: 'It's not important to me to make my own healthcare decision'. This may indicate that making choices is important in some cases; however, it may also reflect the negative wording of the statement.

\section{Strengths and limitations of the study}

This study involved a large-scale survey of patients in primary care to develop a new research tool. The sample size enabled the assessment of the scale's psychometric properties and the high response rate provided some basis for generalisations to the community population as a whole. However, the sample was somewhat skewed towards those who were white and educated to a higher level. Further assessments of validity could be made by comparing scores on the scale with patients' actual decisions about their health care.

\section{Comparison with existing literature}

Policy and research emphasise choice as central to the provision of health care. ${ }^{2}$ Results from this study indicate that beliefs about the value of choice can be measured. This study also offers a new tool for assessing beliefs about having choices and making choices. The positive beliefs shown towards having choices provide support for research that indicates that people prefer choice options over no-choice options and illustrate the 'lure of choice'; ${ }^{10}$ they are also in line with current policy. ${ }^{2}$ Beliefs about making choices were less positive than those related to having choices. Some previous studies point to negative evaluations of choice. ${ }^{1,9}$ It is possible that, in part, this is when the aspect of choice being operationalised relates to making, rather than having, choices.

\section{Funding body}

ESRC award: RES-000-22-1658

\section{Ethical approval}

Ethical approval for this study was awarded by Royal Surrey Ethics Committee (07/Q1909/53)

\section{Competing interests}

The authors have stated that there are none

\section{Acknowledgements}

The authors are grateful for the support from the GPs and receptionists from the four practices, as well as to the patients for completing the questionnaires

\section{Discuss this article}

Contribute and read comments about this article on the Discussion Forum: http://www.rcgp.org.uk/bjgp-discuss

\section{REFERENCES}

1. Schwartz B. The paradox of choice: why more is less. New York: HarperCollins, 2004.

2. Coulter A. Paternalism or partnership? Patients have grown up and there's no going back. BMJ 1999; 319(7212): 719-720.

3. Loewenstein G. Is more choice always better? Social security brief 7 , 1-7. Washington DC: National Academy of Social Insurance, 1999: $1-8$.

4. Botti S, Iyengar SS. The dark side of choice: when choice impairs social welfare. J Public Policy Mark 2006; 25(1): 24-38.

5. Deci EL. Intrinsic motivation. New York: Plenum Press, 1975.

6. Deci EL, Ryan RM. Intrinsic motivation and self determination in human behaviour. New York: Plenum Press, 1985.

7. Glass DC, Singer JE. Urban stress: experiments on noise and social stressors. New York: Academic Press, 1972.

8. Taylor SE. Positive illusions: creative self-deception and the healthy mind. New York: Basic Books, 1989.

9. Iyengar SS, Lepper MR. When choice is demotivating: can one desire too much of a good thing? J Pers Soc Psychol 2000; 79(6): 995-1006.

10. Bown NJ, Read D, Summers B. The lure of choice. J Behav Decis

11. Iyengar SS, Lepper MR. Rethinking the value of choice: a cultural perspective on intrinsic motivation. J Pers Soc Psychol 1999; 76(3): 349-366.

12. Ogden J, Daniells E, Barnett J. Reconceptualising choice: when is choice a good or bad thing? Psychol Health Med. In press.

13. Barnett J, Ogden J, Daniells E. The value of choice: a qualitative study. Br J Gen Pract 2008; 58(554): 609-613. Mak 2003; 16(4): 297-308. 